\title{
Relativistic Quantitative Determination of the "Mysterious" Differences in the Hubble Constant
}

\author{
Ernst Karl Kunst \\ Königswinter, Germany \\ Correspondence to: Ernst Karl Kunst, ernstkunst@aol.com
}

Keywords: Hubble Constant, Different Measurement Results, Earthbound and Space-Telescopes, Different Orbits, Different Hubble Constants; Symmetric Special Relativistic Origin

Received: November 12, $2020 \quad$ Accepted: January 18, $2021 \quad$ Published: January 21, 2021

Copyright $\odot 2021$ by author(s) and Scientific Research Publishing Inc.

This work is licensed under the Creative Commons Attribution International License (CC BY 4.0).

http://creativecommons.org/licenses/by/4.0/

\section{(c) (i) Open Access}

\section{ABSTRACT}

This paper discusses the "Hubble constant measurement-mystery". Independent measurements of this cosmic parameter, referred to as $H_{0}$ in abbreviated form, have all led to different values, with the highest value $\approx 74 \mathrm{~km} \cdot \mathrm{s}^{-1} \cdot \mathrm{Mpc}^{-1}$ and the lowest $\approx 67 \mathrm{~km} \cdot \mathrm{s}^{-1} \cdot \mathrm{Mpc}^{-1}$, where $\mathrm{km}$ denotes kilometer, s second and $\mathrm{Mpc}^{-1}$ megaparsec. These measurements have mainly been obtained with space telescopes. Apparently, up to now there was no way to explain the differences. However, previously published studies seem to regard the problem of the different measurement results for $H_{0}[1,2]$. I have shown that due to a symmetrical expansion of the Minkowski space (SMS), each respective frame of reference for an observer, who rests in the zero point of the frame, is converted into a state of apparent rest relative to the cosmic microwave background (CMB) radiation. This SMS-relativistic effect also seems to be responsible for the different measurement results of the Hubble constant, especially through space telescopes.

\section{INTRODUCTION}

As well known, Georges Lemaitre and Edwin Hubble proposed at the end of the twenties of the last century a redshift of distant galaxies, indicating their withdrawal with growing distance, which is proportional to their distance (to a high degree in the "nearer" neighborhood of the Milky Way Galaxy) [3, 4]. The "Hubble-Lemaitre relationship" $H_{0}$ since is considered as due to the isotropic expansion of the Universe, and thus as the main observational pillar for the current standard cosmological model. Though, the idea of an expanding space-time on the grounds of the field equations of Einstein's General Relativity Theory has been developed first by the Russian Mathematician Alexander Friedmann already in 1922 [5]. In the local universe $H_{0}$ in reality is the proportionality parameter of the (approximate) linear relationship between galaxy distances " $r$ " and the assumed cosmic expansion velocity " $V$ ", derived from the redshift of their spectra: $H_{0}=V / r=\mathrm{km} \cdot \mathrm{s}^{-1} \cdot \mathrm{Mpc}^{-1}$. In the modern full formula still some other parameters, such as 
"dark energy" and "dark mass", are taken into account. But this is of no relevance to the special relativistic solution proposed below for the Hubble puzzle. Pay particular attention, that velocity " $V$ " is precisely known from the exactly measured spectra, whereas distance " $r$ " and the assumed other parameters can be estimated more or less accurately only.

Essentially two methods of measuring $H_{0}$ existed until recently. The first uses the CMB radiation in the surrounding space, so to speak, and tracks the expansion rate in the early universe to what it ought to be now, according to the standard model of cosmology. The second method bases on the so called "distance ladder". The latter relies e.g. on stars called Cepheid variables, which fluctuate in brightness at a rate related to their absolute luminosity. This period-luminosity connection was discovered by the astronomer Henrietta Leavitt at the beginning of the last century and for the first time calibrated on the Cepheid stars of the small Magellanic Cloud [6]. Knowing the brightness of those variables enables astronomers to determine their distance, which they then can use to determine the distances to Cepheids located in farther galaxies. The same method is used by replacing the Cepheids by supernovae Ia, whose luminous behavior is also very well known, and, very recently, by stars called the tip of the red giant branch, e.g. located in the plane of our galaxy and in the halo of other galaxies, too.

In summary: According to astronomical understanding, the apparent luminosity of the cosmic objects of the same type mentioned, expresses their distance from us. From this, after absolute calibration of their brightness, there is the possibility of assigning a clear cosmic distance to these objects and thus to derive the Hubble constant with the help of the "distance ladder". Note, that the most fundamental part of the distance ladder, the first stage, in modern times was measured almost exclusively by space telescopes, orbiting Earth, some point of the Earth orbit or beyond Earth in the ecliptic plane, with some exceptions: e.g. the 2.5-m wide-angle optical telescope (SDSS) at Apache Point Observatory in New Mexico, USA, the Atacama Cosmology six-meter (radio) Telescope (ACT) and the 4-meter Victor M. Blanco Telescope located at Cerro Tololo Inter-American Observatory (CTIO). The latter two are located in Chile. Furthermore, that the "best" estimate of $H_{0} \approx 67 \mathrm{~km} \cdot \mathrm{s}^{-1} \cdot \mathrm{Mpc}^{-1}$, based on repeated measurements from the "local" $\mathrm{CMB}$ radiation by the Planck satellite, was confirmed by measurements from the earthbound SDSS, ACT-radio telescope and the CTIO.

\section{THE INCOMPATIBLE MEASUREMENTS OF VARIOUS SPACE TELESCOPES (ST) AND THE THREE EARTHBOUND TELESCOPES}

When evaluating the measurements of the Hubble constant published in the last twenty years, it is apparent that the former results group around three or four numerical values, namely $H_{0} \approx 67,69-70,71$ 72 and $73-74 \mathrm{~km} \cdot \mathrm{s}^{-1} \cdot \mathrm{Mpc}^{-1}$. The most measurement data resulting in a varying Hubble constant mainly come from the Hubble ST and partly also from other ST, e.g. the GAIA ST, while the Planck satellite has delivered consistent results for the research team of the same name for ten years now. Thereby, as already mentioned, the Planck ST is considered providing the most accurate results to date, which with $H_{0} \approx 67$ $\mathrm{km} \cdot \mathrm{s}^{-1} \cdot \mathrm{Mpc}^{-1}$ have remained practically constant since its launch in May 2009, on this cosmological parameter from CMB observations. In the following we adopt the value $H_{0}=67.4 \pm 0.5 \mathrm{~km} \cdot \mathrm{s}^{-1} \cdot \mathrm{Mpc}^{-1}$ from the 2018 publication of the Planck collaboration as a starting value for the calculation of the other measured $\mathrm{H}_{0}$ values below [7]. The three earthbound telescopes SDSS, ACT and CTIO delivered with $H_{0}=67.6 \pm 1.3$, $67.9 \pm 1.5$ and $67.8 \pm 1.3 \mathrm{~km} \cdot \mathrm{s}^{-1} \cdot \mathrm{Mpc}^{-1}$ about the same result [8] [9] [10]. Note that in the last four cases observation and evaluation took place in one and the same rest system: the Planck satellite, which circles a point on the Earth's orbit, and in the SDSS respectively ACT and CTIO observatory, which earth-based circle the sun. Because according to symmetric SRT is valid [11]:

$\Delta x(\Delta t)^{-1}=\Delta x^{\prime}\left(\Delta t^{\prime}\right)^{-1}=\Delta x \gamma_{0}^{\prime}\left(\Delta t \gamma_{0}^{\prime}\right)^{-1}=\Delta x^{\prime \prime}\left(\Delta t^{\prime \prime}\right)^{-1}=\Delta x \gamma_{0}^{\prime \prime}\left(\Delta t \gamma_{0}^{\prime \prime}\right)^{-1}=c \quad$ and $\quad \Delta x^{\prime}(\Delta t)^{-1}=\Delta x \gamma_{0}^{\prime}(\Delta t)^{-1}=c \gamma_{0}^{\prime}$ $\left(\gamma_{0}\right.$ denotes the respective Lorentz factor) etc., the Planck satellite and the three observatories must during measurements and subsequent data evaluations (observer in the resting system) be regarded as resting relative to the CMB. In all other cases the ST in question, while moving relative to the $\mathrm{CMB}$, only provided observations for the observer (observatory) on Earth, resting in his or her system at rest relative to the 
$\mathrm{CMB}$, for further evaluation. It is clear that the respective rotational speed in relation to the average speed of approximately 370 respectively $340 \mathrm{~km} \cdot \mathrm{s}^{-1}$ (see below) of the Earth relative to the CMB can be completely neglected.

The most controversial difference between the astrophysical data from the Planck ST, the SDSS, the ATC, the CTIO and all other ST is in the discrepancy with the distance-ladder measurements of $H_{0}$. The values measured with Cepheid stars and Supernovae by this method differ from the measurements of the Planck satellite, the SDSS, the ATC and the CTIO. While the values of the latter are consistent with the current understanding of the cosmos, the values measured for the local universe by the Hubble ST and other ST contradict this accepted theoretical model of the universe. The experts agree that all measurements indicate a systematic difference between the values for the Hubble constant obtained directly from the distance to local or medium-sized sources and the values derived indirectly from the CMB radiation. But independent tests show that this discrepancy is not due to physical or measurement errors.

Notice, that by using the Hubble ST for observations of Cepheids in the Large Magellanic Cloud (LMC), to calibrate the first step of the distance ladder, researchers found for the Hubble constant the value $H_{0}=74.22 \pm 1.82 \mathrm{~km} \cdot \mathrm{s}^{-1} \cdot \mathrm{Mpc}^{-1}$ [12]. However, other researchers published the results of a further, additional measurement of the Hubble constant, likewise from the Hubble ST, with $H_{0}=69.8 \pm 1.9$ $\mathrm{km} \cdot \mathrm{s}^{-1} \cdot \mathrm{Mpc}^{-1}$ also by means of the cosmic distance ladder [13]. This measurement has been based on stars, called the tip of the red giant branch, located in the plane of the Milky Way. It has been found that the luminosity of these stars reaches a stable state with increasing age, i.e. they become not brighter and shine all with approximately the same luminosity. This method also delivered a completely different result, namely $H_{0}=72.4 \pm 2.0 \mathrm{~km} \cdot \mathrm{s}^{-1} \cdot \mathrm{Mpc}^{-1}$, when another research team used the observation data of the Hubble ST from red giant stars of the LMC instead of the Milky Way to calibrate the distance ladder [14]. And the very latest value $H_{0}=73.3 \pm 3.1 \mathrm{~km} \cdot \mathrm{s}^{-1} \cdot \mathrm{Mpc}^{-1}$ has been delivered also by the Hubble ST by directly looking at the gravitational lensing effects of quasars [15]. With this new method the Schwarzschild field of the foreground cosmic object acts like a giant magnifying lens, amplifying and distorting light from background objects. Therefore it seems to be completely independent of the "distance ladder", but it is shown below that this is not the case. In the following also will be shown that all measurement results are correct and that the varying $H_{0}$ values are due to the SMS-relativistic expansion of the respective ST's orbit.

\section{DIFFERENT MEASUREMENT RESULTS OF THE HUBBLE CONSTANT $\boldsymbol{H}_{0}$ DUE TO DIFFERENT SMS-RELATIVISTIC EXTENSION OF THE PARALLACTIC ORBIT-LENGTH AND THE ASSOCIATED SHORTENING OF THE DISTANCE}

In reference [11] an extension of the space-time of special relativity to the symmetric Minkowski space-time (SMS) has been introduced, with a relative frame of reference of nature $\Sigma_{0}$ between any two translationally moving frames of reference and an absolute rest frame of nature $\Sigma_{00}$ in the form of the space-fabric of the SMS, indicated through the CMB radiation. The theory also predicts that in the case of one-way movements the time in the moving system appears to be stretched by a factor of $\gamma^{-2}$, where $\gamma$ denotes Lorentz-factor. On these theoretical grounds, among others, the cause of the discrepancies between the measurements of the HIPPARCOS satellite and earthbound measurements of the Pleiades distance could be attributed in ref. [1] to the speed of the satellite of approximately $370 \mathrm{~km} \cdot \mathrm{s}^{-1}$ relative to the earthly lab, which is at rest relative to the $\mathrm{CMB}$, according to the equations:

$$
\begin{aligned}
\Delta x_{(1-\mathrm{way})} & =370 \times \Delta T_{(1-\mathrm{way})} \\
& =370 \times \frac{\Delta x_{01(v \top) 1 \text {-way }}-\Delta x_{01(1-\mathrm{way})}}{2 \times 370 \times \Delta t_{01}} \times 133.5 \mathrm{pc}, \\
\Delta x_{1-\mathrm{way}(133.5 \mathrm{pc})} & =\left[\left(1-\frac{370^{2}}{c^{2}}\right)^{-1}-1\right] \times \frac{370^{2}}{2} \times 133.5 \mathrm{pc}=13.92 \mathrm{pc},
\end{aligned}
$$


where $\Delta x_{1-w a y(133.5 \mathrm{pc})}$ denotes the difference between the distance measured from Earth of $135 \mathrm{pc}$ and the ST moved relative to the CMB. The result Equation (2) is the distance to which, according to the HIPPARCOS measurements, the Pleiades seem to be closer to Earth in comparison to conventional terrestrial measurements, whereby the number two in the denominator of the latter equations is due to the location of the Pleiades cluster close to the ecliptic in both the northern and southern hemispheres, reflecting Earth's annual motion nearly as a line. The above equations are also valid at the quantum level and explain among others also the enigma of the changing proton charge radius, depending on whether the atom is occupied by electrons or muons [2]. In the following it is shown that the SMS-relativistic effect according to Equations (1) and (2) is also responsible for the different measurements of the Hubble constant.

So far, the results of several ST measurements are available. We limit ourselves to the discrepancies in the results of the Hubble ST on the one hand (moving relative to the CMB), and the Planck ST, respectively the SDSS ATC and CTIO on the other hand (resting relative to the CMB), because in them the special-relativistic cause becomes fully visible. As already remarked, are the measurement results of the Planck ST the only ones solely derived by an ST from the CMB power spectrum in the solar vicinity and, therefore, not expected to be altered by the above introduced special relativistic effects. The same is true for the results from the earthbound SDSS, ATC and CTIO. As already mentioned, in the four cases the measurements according to the SMS-extension of special relativity have been executed in the rest-frame at rest of the respective observer. While all other measurement results, and the Hubble constant derived from them, without exception stem from STs, which are either orbiting Earth (e.g. Hubble ST) or certain points in Earth's orbit (e.g. Gaia ST). From the respective relativistic extended orbit the correspondingly shortened distance of "relatively" near cosmic objects (e.g. Cepheids in the Magellanic Clouds) has been determined and then extrapolated into the greatest cosmic distances (cosmic distance ladder) by luminosity comparison. That is why the Planck- and SDSS respectively ATC and CTIO results, and the Hubble constant derived from them, are considered in the following as the only correct, unadulterated measurement results, from which the other relativistic altered results can be calculated according to our theory. In the case of the Hubble ST should be remarked, that according to astronomical data, the ecliptic plane and thus the Earth's orbit is inclined by $\approx 63^{\circ}$ against the plane of the Milky Way, against which the polar axis of the Earth is inclined by $23.44^{\circ}$. The Hubble ST in turn orbits Earth at an inclination angle of $28.5^{\circ}$. These orbital elements add up in such a way that the plane of the Hubble orbit is almost perpendicular to the plane of the Milky Way and roughly in line with the Magellanic Clouds (see below).

As already stated above, is the Hubble parameter $H_{0}=v / r=\mathrm{km} \cdot \mathrm{s}^{-1} \cdot \mathrm{Mpc}^{-1}$ the proportionality constant of the linear relationship between galaxy distances $r$ and the velocity $v$ of the assumed cosmic expansion. Only velocity $v$ is precisely known to Astronomers from the exactly measured spectra. Therefrom in the case of relativistic changes, i.e. shortening of the distance $r$, follows $H_{0} r=H_{0}^{\prime} r^{\prime}=v=$ const. Because always $H_{0}^{\prime}>H_{0} \Rightarrow r_{0}^{\prime}<r_{0}$, follows immediately:

$$
\frac{H_{0} r_{0}}{H_{0}^{\prime} r_{0}^{\prime}}=\frac{H_{0} r_{0}}{H_{0}^{\prime}\left(r_{0}-\Delta r_{0}\right)}=1, H_{0}^{\prime}=\frac{H_{0} r_{0}}{r_{0}-\Delta r_{0}}=\frac{H_{0}}{1-\frac{\Delta r_{0}}{r_{0}^{*}}}, r_{0}^{*}=1+\Delta r_{0} .
$$

It is clear that (according to the following Equation (4)) the respective $\Delta r_{0}^{\prime}-\Delta r_{0}^{\prime \prime \prime}$ (see Equations (5), (7) and (9) below) only refer to the respective relativistic increased distance $r_{0}^{*}=1+\Delta r_{0}$, because if $\Delta r_{0} \rightarrow \infty \Rightarrow v \rightarrow c$. According to the right-hand side of Equation (1), the former's distance traveled will be enlarged by the difference:

$$
\Delta r_{0}=370 \times \Delta T_{(1-\text { way })}=370 \times \frac{\Delta x_{01(v \top) 1-\text { way }}-\Delta x_{01(1-\mathrm{way})}}{2 \times 370 \times \Delta t_{01}} \times r_{0} .
$$

We start from the already well-founded assumption that the final 2018 value $H_{0}=67.4 \pm 0.5$ $\mathrm{km} \cdot \mathrm{s}^{-1} \cdot \mathrm{Mpc}^{-1}$, delivered by the Planck ST (and practically the same value from the SDSS, the ATC and the 
CTIO telescopes) for the Hubble constant represents the correct measurement result in this solar system, not altered by special relativistic or unknown cosmic effects. Thus, in this case, when the ST's movement relative to the absolute rest-frame of nature or the CMB is revealed as the relativistic extension $\Delta r$, related to the number one as a unit because $\Delta r_{x} / r_{x}=\Delta r_{1} / 1$ (where $x$ is any number), one calculates:

$$
\begin{aligned}
& \Delta r_{0}^{\prime}=\left[\left(1-\frac{370^{2}}{c^{2}}\right)^{-1}-1\right] \times \frac{370^{2}}{2} \times 1=0.104244, \\
& r_{0}^{\prime}=1+0.104244=1.104244
\end{aligned}
$$

Therefore, the greatest possible relativistic extension of the Hubble constant according to equation (3) takes the value:

$$
H_{0}^{\prime}=\frac{H_{0}}{\left(1-\frac{\Delta r_{0}}{r_{0}^{\prime}}\right)}=\frac{67.46}{\left(1-\frac{0.104244}{1.104244}\right)}=74.426046 \mathrm{~km} \cdot \mathrm{s}^{-1} \cdot \mathrm{Mpc}^{-1}
$$

That is, the parallactic orbit-length of the Hubble ST appears to be extended by this amount (5) and, thus, the distance to the Magellanic Clouds shortened accordingly. This means that this reduction in distance is transferred to the distance $r$ in the Hubble relation via the "distance ladder" and thus, due to $v=$ constant, a corresponding increase in $H_{0}$ is achieved. The same is true in those cases, where the ST's orbit is other inclined relative to the same observation object than the Hubble ST. From Equation (4) follows in the event that the object observed by the ST in question is approximately in the sky between its orbital plane and the pole of the latter:

$$
\Delta r_{0}^{\prime \prime}=\frac{0.104244}{\pi} \times 2=0.066364, r_{0}^{\prime \prime}=1+0.066364=1.066364
$$

And so the second smallest relativistic Hubble constant needs to take the value

$$
H_{0}^{\prime \prime}=\frac{H_{0}}{\left(1-\frac{\Delta r_{0}}{r_{0}^{\prime \prime}}\right)}=\frac{67.46}{\left(1-\frac{0.066364}{1.066364}\right)}=71.936915 \mathrm{~km} \cdot \mathrm{s}^{-1} \cdot \mathrm{Mpc}^{-1}
$$

Lastly, we consider the case where the observed object is approximately at the zenith of the satellite orbit. This is true for Hubble measurements of the brightness variations of the light from distant galaxies, which is deflected by gravitational lenses, further, of distances to red giant stars in other galaxies, calculated using the tip of the red-giant branch stars in the galactic plane for the distance ladder. For this one computes from Equation (5):

$$
\Delta r_{0}^{\prime \prime \prime}=\frac{0.104244}{\pi}=0.033182, r_{0}^{\prime \prime \prime}=1+0.033182=1.033182
$$

This delivers the smallest relativistic extension of the Hubble constant in the solar system

$$
H_{0}^{\prime \prime \prime}=\frac{H_{0}}{\left(1-\frac{\Delta r_{0}}{r_{0}^{\prime \prime \prime}}\right)}=\frac{67.46}{\left(1-\frac{0.033182}{1.033182}\right)}=69.69846 \mathrm{~km} \cdot \mathrm{s}^{-1} \cdot \mathrm{Mpc}^{-1}
$$

and corresponds very well with the value $H_{0}=69.8 \pm 0.8 \mathrm{~km} \cdot \mathrm{s}^{-1} \cdot \mathrm{Mpc}^{-1}$, which was measured by the Hubble ST in the cases mentioned.

But one should notice that the measured values of $H_{0}$ also can slightly vary, depending on whether the observing ST is moving about parallel or anti-parallel to the vector of $V_{0(\mathrm{CMB})} \approx 370 \mathrm{~km} \cdot \mathrm{s}^{-1}$ of the Sun relative to $\Sigma_{00}$. This means, if the ST at the time of the measurement of the Hubble constant is moving antiparallel to the velocity vector of $V_{0(\mathrm{CMB})} \approx 370 \mathrm{~km} \cdot \mathrm{s}^{-1}$, i.e., with $V_{0(\mathrm{CMB})} \approx 340 \mathrm{~km} \cdot \mathrm{s}^{-1}$ relative to the $\mathrm{CMB}$, then 
from Equations (4) to (10) in two cases smaller values of $H_{0}$ follow, namely 73.39 instead of 74.43 and 69.35 instead of $71.94 \mathrm{~km} \cdot \mathrm{s}^{-1} \cdot \mathrm{Mpc}^{-1}$. This probably is the cause of the observed slightly differing measurement results of the Hubble constant by some research teams using the same ST. Whereas the Planck, the SDSS, the ATC and the CTIO result $H_{0} \approx 67 \mathrm{~km} \cdot \mathrm{s}^{-1} \cdot \mathrm{Mpc}^{-1}$ is completely independent of the speed relative to the $\mathrm{CMB}$, because the respective observation system must be regarded as resting relative to the latter, as shown above.

\section{CONCLUDING REMARKS}

The last eight relationships Equations (3) to (10) are a complete expression of the laws according to which the relativistic enlarged values of the Hubble constant can be determined from the only correct basic value $\approx 67 \mathrm{~km} \cdot \mathrm{s}^{-1} \cdot \mathrm{Mpc}^{-1}$, taking into account the average speed of the respective ST relative to the CMB and the plane of its orbit relative to the object under observation. Furthermore, it should be noted that the "experimentum crucis" for the theory, presented here, already exists with the different results of the value of the Hubble constant by the same ST and unchanged orbit (Hubble ST). These results are $H_{0}=74.22 \pm$ $1.82 \mathrm{~km} \cdot \mathrm{s}^{-1} \cdot \mathrm{Mpc}^{-1}$ on the basis of luminosity-measurements on Cepheids in the Magellanic Clouds, on the one hand, and $H_{0}=69.8 \pm 0.8 \mathrm{~km} \cdot \mathrm{s}^{-1} \cdot \mathrm{Mpc}^{-1}$ on the basis of red giants in the galactic plane, on the other hand, and again $H_{0} \approx 74 H_{0} \mathrm{~km} \cdot \mathrm{s}^{-1} \cdot \mathrm{Mpc}^{-1}$ (correct value $=72.4 \pm 2.0$ ), when another research team also used the observation data of the Hubble ST from red giant stars of the LMC (instead of the Milky Way), as introduced in ref. [12,13] and [14]. This also applies to the equality of the measurement results, namely $H_{0}$ $\approx 67 \mathrm{~km} \cdot \mathrm{s}^{-1} \cdot \mathrm{Mpc}^{-1}$, of the orbiting Planck satellite and the earthbound SDSS, ATC and CTIO telescopes, as detailed above. Hence, symmetric special relativity seems to fully resolve the discrepancies in the Hubble constant measurement results. In conclusion, it should be pointed out that the logically-physically inferred symmetrical extension of the SRT [11] not only satisfactorily explains the various measurement results for the Hubble constant. As already mentioned, in particular the so called "Proton-radius puzzle" and the cause of the discrepancies between the HIPPARCOS-satellite and earthbound VLBI Pleiades distance measurements could be traced back to the same relativistic cause as the Hubble-constant riddle. Thus, in a variation of a remark by Minkowski, it can be said: the true symmetrical core of the principle of relativity, which was initiated by Lorentz and Fitzgerald and worked out by Einstein, Poincaré and Minkowski, now completely comes to light [16].

Summed up, it is clear that a distance ladder on the grounds of ST observations will always lead to relativistic altered, incorrect results concerning distances of cosmic objects and, therewith, of the Hubble constant. The same is true for direct ST-observations of far cosmic objects, as e.g. gravitational lenses, to avoid the distance ladder, since $k \Delta d+k(d-\Delta d)=k d$. Thereby $d$ denotes distance to a cosmic object, $\Delta d$ the first step of the distance ladder and $k$ the factor causing the relativistic extension of the parallactic orbit-length and the associated shortening of the distance. Finally, it remains to be stated that the so-called "dark energy", which was introduced to interpret the discrepancy of $\approx 67-74 \mathrm{~km} \cdot \mathrm{s}^{-1} \cdot \mathrm{Mpc}^{-1}$ in the measurement results for $H_{0}$ as an accelerated expansion of the cosmos, is proving to be a physical chimera. And there is also no additional cosmic problem, as e.g. recently hypothesized by introduction of a local "Hubble Bubble" [17].

\section{CONFLICTS OF INTEREST}

The author declares no conflicts of interest regarding the publication of this paper.

\section{REFERENCES}

1. Kunst, E.K. (2018) On the Common Relativistic Origin of the Neutron Lifetime Discrepancy, the Slight Superluminality of Neutrinos at Fermilab, and Several Astrophysical Problems. Physics Essays, 31, 219-224. https://doi.org/10.4006/0836-1398-31.2.219

2. Kunst, E.K. (2019) Relativistic Resolution of the "Proton Radius Puzzle" in Association with, the Physical Origin 
of the Proton Rest Mass and Time. Physics Essays, 32, 184-189. https://doi.org/10.4006/0836-1398-32.2.184

3. Lemaître, G. (1927) Un Univers homogène de masse constante et de rayon croissant rendant compte de la vitesse radiale des nébuleuses extra-galactiques. Annales de la Sciétés cientifique de Bruxelles, Sèrie A, 47, 49.

4. Hubble, E.P. (1929) A Relation between Distance and Radial Velocity among Extra-Galactic Nebulae. Proceedings of the National Academy of Sciences, 15, 168-173. https://doi.org/10.1073/pnas.15.3.168

5. Friedmann, A. (1922) Über die Krümmung des Raumes. Zeitschrift für Physik, 10, 377-386. https://doi.org/10.1007/BF01332580

6. Leavitt, S., Pickering, H. and Edward, C. (1912) Periods of 25 Variable Stars in the Small Magellanic Cloud. Harvard College Observatory Circular, Vol. 173, 1-3.

7. Planck Collaboration (2018) 2018 Results. VI. Cosmological Parameters.

8. Grieb, J.N., et al. (2017) The Clustering of Galaxies in the Completed SDSS III Baryon Oscillation Spectroscopic Survey: Cosmological Implications of the Fourier Space Wedge of the Final Sample. MNRAS, 467, 2085-2112.

9. Aiola, S., et al. (2020) The Atacama Cosmology Telescope: DR4 Maps and Cosmological Parameters. Journal of Cosmology and Astroparticle Physics, 12, 47.

10. Macaulay, E., et al. (2000) First Cosmological Results Using Type Ia Supernovae from the Dark Energy Survey: Measurement of the Hubble Constant.

11. Kunst, E.K. (2014) Is the Kinematics of Special Relativity Incomplete? Natural Science, 6, 226-247. https://doi.org/10.4236/ns.2014.64027

12. Riess, A.G., Casertano, S., Yuan, W., Macri, L.M. and Scolnic (2019) Large Magellanic Cloud Cepheid Standards Provide a 1\% Foundation for the Determination of the Hubble Constant and Stronger Evidence for Physics beyond Lambda CDM. The Astrophysical Journal, 876, 85. https://doi.org/10.3847/1538-4357/ab1422

13. Yuan, W.L., et al. (2019) Consistent Calibration of the Tip of the Red Giant Branch in the Large Magellanic Cloud on the Hubble Space Telescope Photometric System and a Re-Determination of the Hubble Constant. The Astrophysical Journal, 886, 61.

14. Freedman, W.L., et al. (2019) The Carnegie-Chicago Hubble Program. VIII. An Independent Determination of the Hubble Constant Based on the Tip of the Rend Giant Branch. The Astrophysical Journal, 882, 34.

15. Keir, B., et al. (2020) Could Quasar Lensing Time Delays Hint to a Core Component in Halos, Instead of $\mathrm{H}_{0}$ Tension? The Astrophysical Journal Letters, 892, L27. https://doi.org/10.3847/2041-8213/ab8012

16. Minkowski, H. (1908) Raum und Zeit. Teubner 1982, Stuttgart, 54-66.

17. Lombriser, L. (2020) Consistency of the Local Hubble Constant with the Cosmic Microwave Background. Physics Letters B, 803, Article ID: 135303. https://doi.org/10.1016/j.physletb.2020.135303 